\title{
Rapport d'étape
}

\section{Améliorer le Portail canadien des pratiques exemplaires}

\author{
N. Sims-Jones, M. Sc. inf. (1); E. Dyke, M.A. (2)
}

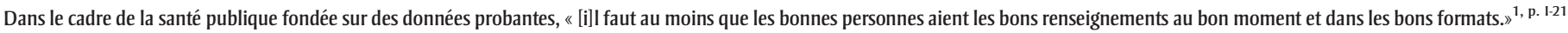

La mission de l'Agence de la santé publique du Canada (ASPC) est de : "promouvoir et protéger la santé des Canadiens au moyen du leadership, de partenariats, de l'innovation et de la prise de mesures dans le domaine de la santé publique $»^{2}$. Afin de contribuer à cette mission, l'ASPC a mis en œuvre de nombreuses initiatives de mobilisation des connaissances en vue d'appuyer la prise de décisions fondées sur des données probantes en santé publique. L'une de ces initiatives, lancées en 2006, est le Portail canadien des pratiques exemplaires $^{3,4}$, une base de données interrogeable en ligne qui contient le détail d'interventions communautaires efficaces visant à promouvoir la santé et à prévenir les maladies chroniques : http://cbpp-pcpe. phac-aspc.gc.ca/fr/.

La conception du Portail s'est inscrite dans une initiative fédérale plus globale, qui comprenait la création de l'ASPC ellemême, visant à renforcer les capacités en santé publique au Canada en réaction au SRAS et aux recommandations du Comité consultatif national sur le SRAS et la santé publique $^{5}$. La nécessité d'établir une base de données de qualité élevée contenant des résumés de données probantes récentes sur l'efficacité des interventions auprès de la population et en santé publique avait été plus particulièrement cernée dans un rapport précédent, Le chemin à parcourir : une consultation pancanadienne sur les priorités en matière de santé publique et des populations ${ }^{6}$.

D’autres initiatives ont également été présentées à cette période pour renforcer les capacités en santé publique. En 2005, six Centres de collaboration nationale en santé publique ont été établis et financés par l'ASPC. Ces Centres «produisent de la documentation destinée à appuyer les professionnels de santé publique à améliorer leur réponse aux menaces à la santé publique, aux maladies chroniques et aux blessures, aux maladies infectieuses et aux inégalités de santé $»^{7}$. Ils sont axés sur la transposition de données probantes produites par des chercheurs afin qu'elles soient utilisées par les praticiens en santé publique ${ }^{7}$.

Une autre excellente source d'information fondée sur des données probantes pour les praticiens en santé publique est HealthEvidence.ca, un site Internet qui donne accès à des « recensions rigoureuses sur le plan méthodologique et récemment achevées portant sur les interventions en santé publique et en promotion de la santé $»^{8}$. Le but ultime de ce projet est « de faciliter l'adoption et la mise en œuvre de politiques, de programmes et d'interventions efficaces pour soutenir la prise de décision en santé publique à l'échelle locale et régionale dans l'ensemble du Canada ${ }^{8}$.

Les administrations provinciales, territoriales et municipales ainsi que les unités de santé locales au Canada ont mis en place des initiatives de renforcement des capacités en santé publique. Par exemple, la Colombie-Britannique a publié Framework for Core Functions in Public Health ${ }^{9}$ pour orienter les programmes de santé publique de la province, tandis que l'Ontario a établi des normes en santé publique qui " établissent les exigences relatives aux programmes et aux services de santé publique fondamentaux ${ }^{10, p .1}$. Le Québec a également été actif dans plusieurs secteurs, notamment grâce à la publication du Programme national de santé publique 2003-2012 du Québec qui permet de " préciser, du même coup, les services de santé publique accessibles à tous les citoyens et citoyennes du Québec »11, p.1. En 2010, la NouvelleÉcosse a produit Moving Forward: A Commitment to Public Health's Future ${ }^{12}$ qui découle d'un rapport antérieur : The Renewal of Public Health in Nova Scotia: Building a Public Health System to Meet the Needs of Nova Scotians ${ }^{13}$. Les autres provinces et territoires ont pris diverses mesures pour augmenter leur capacité en santé publique après le SRAS.

Malgré les ressources offertes aux praticiens en santé publique, les évaluations du Portail canadien des pratiques exemplaires indiquent que l'accès à une information globale pour appuyer la planification, la mise en œuvre et l'évaluation des programmes de santé publique demeure un sujet de préoccupation. Par conséquent, le personnel de l'ASPC a commencé à planifier le contenu d'un Portail amélioré, tout en conservant le thème original, soit la promotion de la santé et la prévention des maladies chroniques.

Afin de choisir un contenu supplémentaire, l'ASPC a passé en revue les lois et les normes en santé publique des provinces et, le cas échéant, des territoires. Le but de cette analyse était de cerner quels secteurs de la promotion de la santé et de la prévention des maladies chroniques la plupart des ministères de la Santé publique au Canada avaient le mandat d'examiner. Comme on pouvait s'y attendre, ces secteurs étaient la lutte contre le tabagisme, la promotion de l'activité physique et de la saine alimentation, ainsi que des approches 
intégrées en matière de prévention des maladies chroniques. Une fois ces thèmes identifiés, des renseignements pertinents ont été sélectionnés à partir des sites Internet gouvernementaux à l'échelle provinciale, territoriale, fédérale et internationale pour chacun des secteurs, afin d'appuyer l'élaboration des programmes.

Pour les programmes de promotion de la santé et ceux portant sur les facteurs de risques modifiables, l'information fournie dans la section "Sujets de santé publique » du Portail amélioré intègre des données de surveillance, des stratégies, des lignes directrices sur ce qui fonctionne comme des directives, des recommandations et des approches stratégiques, ainsi que des liens vers des examens systématiques pertinents de la documentation de Health-Evidence.ca et de la section « Pratiques exemplaires » du Portail.

La section " Maladies chroniques » du Portail amélioré porte principalement sur la prévention de maladies chroniques précises. Dans cette section, un vaste éventail d'information est fourni sur les facteurs de risque, la surveillance, les stratégies de prévention et les lignes directrices, ainsi que des liens vers des publications clés, des sites provinciaux et territoriaux de prévention des maladies, des outils et des ressources, des projets financés et des examens systématiques pertinents de la documentation de HealthEvidence.ca.

La section " Pratiques exemplaires » offre un accès à la base de données interrogeable en ligne sur les interventions communautaires efficaces tirée de la version d'origine du Portail canadien des pratiques exemplaires. La section « Ressources » répertorie maintenant une liste commentée de ressources canadiennes de qualité portant sur la prise de décisions axées sur des données probantes et sur la planification de programmes de santé publique.

Il est souvent demandé aux ministères de la Santé de commenter les politiques de santé publique de leurs administrations ou d'en élaborer. La section du nouveau Portail sur les questions stratégiques réunit des documents clés sur les politiques canadiennes ou internationales en santé publique, ainsi que des liens vers des ressources et des outils stratégiques. Enfin, la section " Apprendre davantage » fournit une liste complète des fils RSS, des mises à jour par courriel et des listes de distribution des organismes de santé publique.

On prévoit d'élargir ensuite l'éventail des sujets en santé publique, d'ajouter d'autres séries de ressources pour appuyer les programmes en santé publique et de proposer une exploration des sites Internet d’organismes locaux en santé publique. Le Portail canadien des pratiques exemplaires est conçu comme une ressource en constante évolution, avec deux mises à jour par an. Si vous avez des commentaires ou des suggestions sur son contenu ou sur l'approche choisie, veuillez communiquer avec nous à : http://cbpp-pcpe .phac-aspc.gc.ca/fr/contact-us/.

\section{Références}

1. Kiefer L, Frank J, Di Ruggiero E, Dobbins M, Manuel D, Gully PR, Mowat D. Favoriser la prise de décision factuelle au Canada : examen de la nécessité de créer un centre de données de recherche et un réseau de recherche sur la santé publique et les populations au Canada. Can J Public Health. 2005;96(3):I20-I40.

2. Agence de la santé publique du Canada. À propos de l'Agence [Internet]. Ottawa (Ont.) : Agence de la santé publique du Canada; [consultation le 19 sept. 2012]. Consultable en ligne à la page : http://www .phac-aspc.gc.ca/about_apropos/index-fra .php

3. Agence de la santé publique du Canada. Le portail canadien des pratiques exemplaires [Internet]. Ottawa (Ont.) : Gouvernement du Canada; [consultation le 19 sept. 2012]. Consultable en ligne à la page : http: //cbpp-pcpe.phac-aspc.gc.ca

4. Finkle-Perazzo D, Jetha N. Forum pancanadien - Ressources en ligne pour améliorer la prise de décision en matière de santé publique. Maladies chroniques et blessures au Canada, 2011;31(4):177-181.
5. Comité consultatif national sur le SRAS et la santé publique. Leçon de la crise du SARS : renouvellement de la santé publique au Canada. Santé Canada; oct. 2003 [consultation le 19 sept. 2012]. Consultable en ligne à la page : http://www.phac-aspc .gc.ca/publicat/sars-sras/naylor/index-fra .php

6. Institut de la santé publique et des populations, Initiative sur la santé de la population canadienne. Le chemin à parcourir : une consultation pancanadienne sur les priorités en matière de santé publique et des populations [Internet]. Ottawa (Ont.) : ICIS; 2002 [consultation le 19 sept. 2012]. PDF (843 Ko) téléchargeable à partir du lien : http://www.cihr-irsc.gc.ca/f/documents /charting_the_course_f.pdf

7. Centres de collaboration nationale en santé publique. À propos des CCNSP [Internet]. Centres de collaboration nationale en santé publique; 2009 [consultation le 19 sept. 2012]. Consultable en ligne à la page : http://www.ccnsp.ca/57/e_propos_des _Centres.ccnsp

8. Health-evidence.ca. À propos de nous [Internet]. Hamilton (Ont.) : Health-evidence.ca [consultation le 19 sept. 2012]. Consultable en ligne à la page : http: //www.donneesprobantes-sante.ca/html /AboutUsFR

9. Ministry of Health Services, Population Health and Wellness. A framework for core functions in public health: resource document. Victoria (BC) : BC Ministry of Health Services, Population Health and Wellness; 2005 [consultation le 19 sept. 2012]. PDF (1,35 Mo) téléchargeable à partir du lien : http://www.health.gov.bc.ca/public-health /pdf/core_functions.pdf

10. Ministère de la Santé et des Soins de longue durée de l'Ontario. Normes de santé publique de l'Ontario 2008, Toronto (Ont.) : ministère de la Santé et des Soins de longue durée de l'Ontario; 2008 [consultation le 19 sept. 2012]. PDF (494 Ko) téléchargeable à partir du lien : http://www.health.gov .on.ca/fr/pro/programs/publichealth/oph_ standards/docs/ophs_2008f.pdf 
11. Cardinal L, Francoeur D, Hamel M, Kirouac S, Théberge Y. Programme national de santé publique 2003-2012. Québec (Qc) : Santé et Services sociaux Québec; 2003 [consultation le 19 sept. 2012]. PDF téléchargeable à partir du lien : http://publications.msss.gouv.qc .ca/acrobat/f/documentation/2003/03-21602A.pdf

12. Nova Scotia Public Health. Moving forward: a commitment to public health's future. Halifax (NS) : Nova Scotia Public Health; 2010.

13. Moloughney B. The renewal of public health in Nova Scotia: building a public health system to meet the needs of Nova Scotians. Halifax (NS): Nova Scotia Health Promotion and Protection; 2006 [consultation le 19 sept. 2012]. PDF téléchargeable à partir du lien : http://www.gov.ns.ca/hpp/publications /07148_therenewalofphinnsreport_apr06_en .pdf 